\title{
Adrenocortical and Pituitary Glucocorticoid Feedback in Abstinent Alcohol-Dependent Women
}

\author{
Bryon Adinoff, Susan E. Best, Wen Ye, Mark J. Williams, and Ali Iranmenesh
}

\begin{abstract}
Background: The long-term ingestion of alcohol diminishes hypothalamic-pituitary-adrenal (HPA) axis reactivity in alcohol-dependent men, potentially altering future relapse risk. Although sex differences in HPA axis functioning are apparent in healthy controls, disruptions in this system have received little attention in alcohol-dependent women. In this study, we assessed the basal secretory profile of adrenocorticotropic hormone (ACTH) and cortisol, adrenocortical sensitivity in both the presence and absence of endogenous corticotropic pituitary activation, and feedback pituitary glucocorticoid sensitivity to dexamethasone.

Methods: Seven women 4- to 8-week abstinent alcohol-only dependent subjects and 10 agematched female healthy controls were studied. All subjects were between 30 and 50 years old, not taking oral contraceptives, and were studied during the early follicular phase of their menstrual cycle. Circulating concentrations of ACTH and cortisol were measured in blood samples collected at frequent intervals from 2000 to 0800 hour. A submaximal dose of cosyntropin $(0.01 \mu \mathrm{g} / \mathrm{kg})$, a synthetic ACTH (1-24), was administered at 0800 hour to assess adrenocortical sensitivity. In a separate session, low-dose cosyntropin was also administered following high-dose dexamethasone ( $8 \mathrm{mg}$ intravenous) to assess adrenocortical sensitivity in the relative absence of endogenous ACTH. In addition, the ACTH response to dexamethasone was measured to determine the pituitary glucocorticoid negative feedback. Sessions were 5 days apart, and blood draws were obtained every 5 to 10 minutes.

Results: Mean concentrations and pulsatile characteristics of ACTH and cortisol over 12 hours were not statistically different between the 2 groups. Healthy controls had a somewhat higher $(p<0.08)$ net peak, but not net integrated, cortisol response to cosyntropin relative to the alcohol-dependent women. There were no significant group differences in either the ACTH or cortisol response to dexamethasone nor in the net cortisol response to cosyntropin following dexamethasone.

Conclusion: Significant differences in pituitary-adrenal function were not apparent between alcohol-dependent women and matched controls. Despite the small $n$, it appears that alcoholdependent women do not show the same disruptions in HPA activity as alcohol-dependent men. These findings may have relevance for gender-specific treatment effectiveness.
\end{abstract}

Key Words: Adrenal Cortex, Alcoholism, Cosyntropin, Dexamethasone, Pituitary-Adrenal System, Gender, Female.

A WEALTH OF literature supports disruptions of the hypothalamic-pituitary-adrenal (HPA) axis activity in alcohol-dependent men. Whereas increases in cortisol are observed during intoxication (Adinoff et al., 2003; Mendelson et al., 1971; Stokes, 1973) and withdrawal (Adinoff et al., 1991; Iranmanesh et al., 1989; Keedwell et al., 2001;

From the Department of Psychiatry (BA, SEB, MJW), University of Texas Southwestern Medical Center at Dallas, Dallas, Texas; Veterans Affairs North Texas Health Care System (BA, SEB), Dallas, Texas; Department of Biostatistics (WY), University of Michigan, Ann Arbor, Michigan; Department of Medicine (AI), University of Virginia School of Medicine, Charlottesville, Virginia; and Salem VA Medical Center (AI), Salem, Virginia.

Received for publication May 22, 2009; accepted January 4, 2010.

Reprint requests: Bryon Adinoff, MD, 5323 Harry Hines Blvd., Dallas, TX, 75390-8564; Fax: 214-645-6976; E-mail: bryon.adinoff@, utsouthwestern.edu

Copyright (C) 2010 by the Research Society on Alcoholism.

No claim to original U.S. government works

DOI: 10.1111/j.1530-0277.2010.01164.x
Mendelson et al., 1971), an attenuated adrenocorticoid response to both the behavioral and pharmacological activation of the HPA axis (Adinoff et al., 1998) has been observed during the early weeks of abstinence. Diminished glucocorticoid reactivity has been reported in alcohol-dependent subjects following an alcohol challenge (Merry and Marks, 1972), insulin-induced hypoglycemia (Chalmers et al., 1978; Costa et al., 1996), exercise (Coiro et al., 2007), operative trauma (Margraf et al., 1967), nicotine (Coiro and Vescovi, 1999), corticotropin releasing hormone (CRH) stimulation (Adinoff et al., 2005b; Bailly et al., 1989), cosyntropin stimulation (Adinoff et al., 2005a; Wand and Dobs, 1991), hyperthermia (Vescovi et al., 1997), cold pressor (Errico et al., 1993), mental arithmetic (Errico et al., 1993), and public speaking (Lovallo et al., 2000). The clinical relevance of these findings has been suggested by recent studies demonstrating that attenuated basal glucocorticoid concentrations (Kiefer et al., 2006) or a hyporeactive adrenocortical response (Junghanns et al., 2003) predict prospective relapse. 
These findings, however, have been almost exclusively assessed in alcohol-dependent men. The aforementioned referenced studies measuring pituitary-adrenal responsivity (Chalmers et al., 1978; Coiro et al., 2007; Coiro and Vescovi, 1999; Costa et al., 1996; Ehrenreich et al., 1997; Errico et al., 1993; Junghanns et al., 2003; Knudsen et al., 1987; Lovallo et al., 2000; Merry and Marks, 1972; Vescovi et al., 1997; Wand and Dobs, 1991), for example, consisted of 276 alcohol-dependent men and no alcohol-dependent women. There are several likely reasons accounting for this representational bias. First, there are more male than female alcoholdependent subjects (Kandel et al., 1997), though this clearly would not account for the absence of women in this literature. Second, many studies are conducted in the Veterans Administration hospitals, which treat primarily male subjects. Perhaps more important, however, is the difficulty in correctly studying neuroendocrine parameters in female alcohol-dependent subjects. Women are more likely to suffer from co-morbid psychiatric disorders than their male counterparts (Brady et al., 1993; Najavits et al., 1997), requiring the exclusion of many, if not most, female subjects. Most problematic is that the neuroendocrine system in women should optimally be assessed during a period of relative hormonal stability, requiring a similar hormonal phase of life (i.e., premenopausal) between subjects, a matched (and preferably quiescent) menstrual phase, and absent the use of synthetic hormonal treatment (i.e., oral contraceptives) (Kirschbaum et al., 1999) that may alter HPA axis responsivity. To assess menstrual phase without resorting to the frequent measurement of gonadotropic hormones also requires the presence of a functionally intact uterus and regular menses. Given the irregular menses frequently observed in alcohol-dependent women, particularly during short-term abstinence, these collective difficulties can be daunting.

Nevertheless, stress-hormone reactivity in alcohol-dependent women requires investigation. Because of significant gender differences in HPA axis reactivity to both behavioral and pharmacological stimuli (Shalev et al., 2008; Uhart et al., 2006), coupled with gender-specific genetic modulators (Shalev et al., 2008), findings in men may not generalize to women. Increased rates of stress-related psychiatric disorders in alcohol-dependent women, such as Post-traumatic Stress Disorder (Brady et al., 1993; Najavits et al., 1997) or Major Depressive Disorder, suggest that biological processes underlying stress may also differ in female compared to male alcohol-dependent subjects. Given the role of stress in relapse and the aforementioned putative relationship between hypoadrenocortical reactivity and relapse risk, an understanding of pituitary-adrenal responsiveness takes on increased importance in this vulnerable population. Finally, the finding of adrenocortical hyporeactivity in alcohol-dependent subjects is not universally observed (Adinoff et al., 1990; Anthenelli et al., 2001; Brady et al., 2006; George et al., 1994; Inder et al., 1995; Munro et al., 2005), indicating the need for additional studies in both genders. In addition, few studies have separately assessed the contribution of the adrenal cortex relative to higher-level organs (hypothalamus or pituitary gland) in alcohol-dependent patients.

In this study, we conducted a series of experiments to assess the pituitary-adrenal function in abstinent alcohol-dependent women and compared their results to age-matched healthy controls. All subjects were without a lifetime history of Axis I disorders (other than alcohol-dependence in the patient sample or nicotine dependence in either group), were not taking oral contraceptives, and were studied immediately after the onset of their menses. As previous research suggests that the blunted pituitary-adrenal response in alcohol-dependent subjects is because of an attenuated adrenocorticoid responseeven in the presence of sufficient ACTH stimulation (Adinoff et al., 2005b) - we focused our investigation upon the adrenal cortex. (Pituitary ACTH is secreted in response the release of hypothalamic cCRH. The release of ACTH into the bloodstream then induces the release of cortisol from the adrenal cortex.) Several paradigms, therefore, were performed to assess the integrity of the adrenal cortex. First, basal pulsatile characteristics of ACTH and cortisol were assessed from 2000 to 0800 hour. Because of safety limitations on the amount of blood withdrawn, measures of basal pulsatility were limited to the period of greatest ACTH and cortisol activity (e.g., 2000 to 0800 hour). Second, adrenocorticoid sensitivity was assessed in response to cosyntropin, a synthetic ACTH $\left(\mathrm{ACTH}_{1-24}\right)$. This paradigm allowed us to directly determine responsivity of the adrenal cortex without the confound of concurrent pituitary ACTH stimulation. In order to avoid a maximal glucocorticoid response, or ceiling effect, that could obscure potential group differences, we assessed adrenocortical sensitivity with a very low dose $(0.01 \mu \mathrm{g} / \mathrm{kg})$ of cosyntropin [0.3\% of the clinically recommended dose $(250 \mu \mathrm{g})$ used in the cosyntropin stimulation test in the diagnosis of endocrine disorders]. Even in this procedure, however, group differences in endogenous basal concentrations of ACTH may alter glucocorticoid responsivity. A third paradigm, therefore, assessed adrenocorticoid responsivity in the near absence of endogenous ACTH. Suppression of pituitary ACTH was accomplished by administering high-dose dexamethasone the night prior to cosyntropin administration. Dexamethasone administration also allowed the assessment of negative glucocorticoid feedback upon the pituitary corticotrophs.

Given the paucity of previous studies in this area, we predicted that the alcohol-dependent women would respond comparatively to the alcohol-dependent men. Thus, our original hypothesis was that adrenocortical reactivity would be blunted in alcohol-dependent women, relative to control women, following cosyntropin both with and without dexamethasone pretreatment.

\section{MATERIALS AND METHODS}

Alcohol-Dependent Subjects (see Table 1)

Seven female alcohol-dependent subjects, ranging in age from 30 to $43 \mathrm{y} / \mathrm{o}$, were recruited from patients requesting treatment for 
Table 1. Demographic and Clinical Characteristics of Alcohol-Dependent Subjects and Healthy Controls (mean \pm SD)

\begin{tabular}{|c|c|c|c|c|c|}
\hline & Alcohol-dependent $(n=7)$ & Controls $(n=11)$ & $t$ or $\mathrm{X}^{2}$ & DF & $p$ Value \\
\hline Age & $36.2 \pm 5.5$ & $37.0 \pm 4.9$ & 0.285 & 16 & 0.779 \\
\hline \multicolumn{6}{|l|}{ Race } \\
\hline Asian & 0 & 0 & 3.368 & 2 & 0.186 \\
\hline Caucasian & 6 & 5 & & & \\
\hline African-American & 0 & 3 & & & \\
\hline Hispanic & 1 & 3 & & & \\
\hline \multicolumn{6}{|l|}{ Marital status } \\
\hline Single & 2 & 2 & 10.63 & 3 & 0.014 \\
\hline Married & 0 & 8 & & & \\
\hline Separated & 2 & 0 & & & \\
\hline Divorced & 3 & 1 & & & \\
\hline Employed & 5 & 11 & 3.536 & 1 & 0.060 \\
\hline Education (yrs) & $12.3 \pm 2,0$ & $14.7 \pm 2.2$ & 2.47 & 14 & 0.027 \\
\hline Nicotine dependent (smokers) & 5 & 1 & 7.481 & 1 & 0.006 \\
\hline Alanine aminotransferase $(\mathrm{U} / \mathrm{I})$ & $30.5 \pm 13.3$ & $15.7 \pm 6.2$ & -3.10 & 15 & 0.007 \\
\hline Aspartate aminotransferase (Ú/I) & $26.5 \pm 10.2$ & $18.2 \pm 4.1$ & -2.34 & 15 & 0.033 \\
\hline GGTP & $130.5 \pm 118.6$ & $9.5 \pm 7.8$ & -3.23 & 15 & 0.006 \\
\hline
\end{tabular}

Comparisons between groups were by $t$-test or chi-squared.

alcohol dependence at Homeward Bound, Inc. or the Dallas VA Medical Center. All 7 subjects participated in both study sessions. Patients reported an alcohol intake of at least $80 \mathrm{gms}$ of absolute alcohol on a daily basis for at least 2 weeks prior to the cessation of drinking, and had at least a 10-year history of problematic drinking. Patients with other lifetime DSM-IV Axis I psychiatric disorders (such as anxiety, post-traumatic stress, schizophrenia, or mood disorders) not associated with alcohol use (i.e., present prior to heavy drinking or following at least months abstinence), other Substance Use Disorders (or use of another drug more than once weekly) within the previous 12 months (excluding caffeine or nicotine use disorders), medical disorders (i.e., hypertension, diabetes, chronic pain, or cardiac or pulmonary disorders), or major head trauma were excluded from the study. Exclusion criteria also included use of any medications that may interfere with HPA axis functioning (i.e., psychotropics, antihypertensives, hypoglycemic agents, oral hypoglycemics) within 2 weeks of the study, Beck Depression Inventory (Beck et al., 1979) (BDI) scores above 15 at the time of assessment, or an alanine aminotransferase or aspartate aminotransferase 3.0 times greater than the clinical laboratory's upper limit of normal.

\section{Healthy Controls}

Eleven healthy control women, ranging in age from 30 to 44 years old, were individually age-matched within a 5-year period with alcohol-dependent subjects. Controls reported no lifetime history of any DSM-IV Axis I Disorder (except Nicotine Use Disorders), reported no medical disorders, and were on no medications. Controls with a single first-degree relative or two second-degree relatives with an Axis I disorder were excluded.

\section{Clinical Assessment (See Table 1)}

All subjects obtained a history and physical exam, routine clinical laboratory testing, electrocardiogram, and urine drug screens. Psychiatric and substance use disorders were assessed using the Structured Clinical Interview for DSM IV (SCID)-Lifetime (First et al., 1996). Alcohol-dependent subjects were detoxified from alcohol, and then housed on a residential treatment unit until the studies were initiated. Urine drug screens were obtained 3 times weekly and breathalyzers were obtained whenever the patient left the unit unaccompanied by staff. Separate informed consents from both the University of Texas Southwestern Medical Center and the Dallas VA Medical Center Internal Review Boards (IRBs) were obtained after the study was fully explained, and subjects were financially compensated for their participation. In the patient population, the Drinker Inventory of Consequences - Lifetime Consequences (DrInC-2L) (Miller et al., 1995) was used to assess lifetime severity of alcohol-related problems, and a Time-Line Follow Back (Sobell and Sobell, 1978) (TLFB) was used to assess 1-month, 90-day, 1-year, and lifetime drinking history.

\section{Procedure}

All neuroendocrine studies were performed at the General Clinical Research Center (GCRC) at the University of Texas Southwestern Medical Center. The studies were performed in 2 separate sessions. The initial session was initiated the day after the onset of menses. In order to assess each subject during the early follicular phase of their menstrual cycle, the sessions were separated by 5 days rather than the 1-week interval used in prior studies of male subjects. Session order was balanced. A nicotine patch was placed on all nicotinedependent subjects upon arrival to the GCRC and was continued throughout their hospitalization. All smokers reported smoking more than 10 cigarettes daily and were administered one $21 \mathrm{mg}$ patch every 24 hours.

Session $A$. This study included a 24-hour urine collection for urinary free cortisol, 12-hour sampling for the measurement of plasma ACTH and cortisol secretory dynamics and 2-hour cosyntropin stimulation test. Because of IRB limitations on the amount of blood obtained ( $350 \mathrm{ml}$ over 6 weeks), it was necessary to limit our assessment of circadian pulsatility to a 12-hour period. The interval from 2000 to 0800 hour was chosen because of the increased pulsatility observed during this period. Subjects were brought to the GCRC at 1800 hour on Day 1. A 24-hour urine collection was initiated at 2000 hour on Day 1 for urinary free cortisol and continued until 2000 hour on Day 2. An intravenous catheter was inserted in each arm at 1900 hour on Day 2. Blood sampling (2cc/sample) at 10-minute intervals was initiated at 2000 hour continued through 0800 hour on Day 3. Sampling was obtained manually by nursing staff. Significant efforts were made to avoid waking subjects while asleep; the intravenous port was 6 inches from the subject, allowing blood drawer without subject contact, and a focused light was used to avoid unnecessary illumination. Following the 0800 hour blood draw on Day 3 , cosyntropin $0.01 \mu \mathrm{g} / \mathrm{kg}$ was administered intravenously over one minute. Blood sampling frequency was increased immediately following cosyntropin administration every five minutes through 0900, and then decreased every 10 minutes through 
1000 hour. Intravenous lines were removed following the final 1000 hour blood draw.

Session B. This study included the pituitary and adrenocortical response to dexamethasone and the cosyntropin stimulation test following dexamethasone. Subjects arrived at the GCRC at 2000 hour, and intravenous lines were placed into both arms at 2100 hour. Blood samples for the measurement of ACTH and cortisol were obtained every 10 minutes from 2200 through 2300 hour. Dexamethasone $8 \mathrm{mg}$ in $50 \mathrm{ml}$ of $\mathrm{D}_{5} \mathrm{~W}$ was administered intravenously from 2300 to 2330 hour. Blood sampling was continued from 2300 to 0500 hour, and then restarted at 0700 to 0800 hour every 10 minutes. (Blood sampling was not obtained from 0500 to 0700 hour because of limitations on blood volume.) The cosyntropin test was performed as described in Session A. Cosyntropin was administered at 0800 hour to assure that dexamethasone (administered 9 hours previously) would continue to maximally suppress endogenous ACTH release throughout the cosyntropin paradigm.

\section{Assays}

Plasma concentration of ACTH was measured by immunoradiometric assay (IRMA), using reagents from DiaSorin (Stillwater, Minnesota). This assay has a low-end sensitivity of $1.5 \mathrm{pg} / \mathrm{ml}$, with intra-assay coefficient of variation of 2.5 to $5.4 \%$ in the concentration range of 33 to $773 \mathrm{pg} / \mathrm{ml}$. Inter-assay coefficient of variation is 3.2 to $5.7 \%$ in the concentration range of 8.7 to $257 \mathrm{pg} / \mathrm{ml}$. Serum concentration of cortisol was measured by radioimmunoassay (RIA) using reagents from DiaSorin. This assay has a low-end sensitivity of $0.21 \mu \mathrm{g} / \mathrm{dl}$, with intra-assay coefficient of variation of 6.6 to $7.7 \%$ in the concentration range of 2.9 to $47.1 \mu \mathrm{g} / \mathrm{dl}$. Inter-assay coefficient of variation is 8.8 to $9.8 \%$ in the concentration range of 3.7 to $36.9 \mu \mathrm{g} / \mathrm{dl}$. RIA kits from DiaSorin were used for the measurement of urinary free cortisol.

\section{Statistics}

Demographics. Students $t$-test (interval data) and chi square contingency table analyses (nominal data) were used to compare demographic characteristics of the 2 groups. Items of interest included age, education, employment status, marital status, liver function, and smoking status. Descriptive statistics were used to quantify drinking characteristics of the alcohol-dependent group including years of problem drinking, drinking days ( 90 days prior to drinking cessation and lifetime), and days abstinent at the time of testing. Students $t$-test was used to compare mean scores on the BDI and the DrInC-2L.

Basal ACTH and Cortisol Pulsatile Characteristics. Because there is a circadian rhythm for the 2 hormones of interest, the assumption that all secretion was pulsatile leads to unreasonably long half-lives for ACTH and cortisol, resulting in a poor fit of the calculated pulses to the actual data. Therefore, pulsatile characteristics were assessed by the Smoothing Baseline Pulse Pulses (SBPP) algorithm (Guo et al., 1999), which allows for a changing baseline. In general, missing values were not an issue in these data, but equally spaced observations are required for analysis in pulse detection algorithms. Missing values were handled in the following way: if the concentration at the time point preceding the missing time point was lower than at the point after the missing time point, the missing concentration was replaced with the concentration value of the point preceding the missing time. This was carried out in order to avoid creating the possibility of a pseudo-pulse. Missing values that were part of a decreasing trend were linearly interpolated. For various reasons, some series had to be excluded from the secondary analysis, mainly because they did not show pulsatility. Pulsatile analysis was completed on seven alcohol-dependent and nine control subjects for
ACTH and cortisol analyses. A Student $t$-test was used to compare group means. A brief description of some of summary measures from SBPP is given in the following paragraph:

\section{Total Probability}

The sum of the probabilities of being a pulse at each point.

\section{Adjusted Input}

The sum of input at each time point times the probability of input at each point. It is a weighted total input.

\section{Number of Inputs}

The number of input locations with a probability $>0.5$ SBPP models the probability of input at each time point and we are using the 0.5 cut off to distinguish large input (where input is most likely occurring) from small (unlikely) input locations. This measure allows the comparison of input frequencies between the 2 groups, but is not a measure of pulse number.

\section{Average Baseline}

The mean of the baseline values estimated at each point. SBPP allows for a changing baseline so baseline itself is no longer a parameter.

\section{Mean Amplitude}

The net mean height of pulses after subtracting the changing baseline.

Time $\times$ Group Analysis. A repeated measure analysis of variance (ANOVA) on the mean hormone level over 4 3-hour blocks (i.e., 2000-2250, 2300-0150, 0200-0450, and 0500-0800 hour) was performed in order to incorporate all subjects into the circadian analysis.

Pharmacologic Challenge Studies. Cortisol secretion following cosyntropin was compared between the hours of 0800 and 1000 hour by the area under the curve method (AUC), or net integrated response, and by net peak cortisol for both Studies A and B. The net integrated response was calculated by taking the average hormone concentration between consecutive measurement points, multiplying by the time interval between the points, summing across time, and netting out basal hormone levels multiplied by the total time interval over which measurement occurred (i.e., 120 minutes). Four subjects revealed a negative integrated cortisol response following cosyntropin (without dexamethasone pretreatment) as their final cortisol concentrations decreased below their basal measures. Thus, the integrated cortisol response was also determined over 60 minutes for this study. Basal hormone values were based on mean levels between the hours of 0730 and 0800 . The response to dexamethasone was determined using mean ACTH or cortisol response from 2330 to 0500 hour. For each analysis, Students $t$-test was used to compare group means. Missing values were estimated based on the mean of hormone measures taken directly before and after the missing values. Two subjects (one patient, one control) had several continuous data points missing following dexamethasone (after 0030 hour). Because of the stability of these measures, missing values were estimated as for the single missing measures just described. Because of technical problems, data from one control was included only for Study A and another control was included only for Study B.

Effect Sizes. Effect sizes (assuming an $a=0.05$ and $b=0.80$ ) were determined using DSTPLAN software. 
Correlation Analysis. The relationship between drinking history (i.e., drinks in previous 30 days, lifetime drinks, days abstinent) and smoking history (nicotine pack years) and various neuroendocrine measures [basal cortisol concentration prior to cosyntropin administration (without dexamethasone), integrated cortisol response following cosyntropin, mean $\mathrm{ACTH}$ response to dexamethasone, and integrated cortisol response following cosyntropin with dexamethasone pretreatment] was assessed by (Pearson) correlation.

\section{RESULTS}

\section{Subject Characteristics (see Table 1)}

The 7 alcohol-dependent women were similar in race $(p<0.19)$ but more likely to be unemployed $(p<0.06)$ and smokers $(p<0.008)$ relative to the healthy controls. Alcohol-dependent subjects scored $35.8 \pm 4.7$ on the DrInC and reported $18.1 \pm 17.2$ years drinking. TLFB revealed 49,311.6 $\pm 36,246.4$ lifetime standard drinks and heavy drinking over the 90 days prior to admission $(1253.1 \pm 477.0$ drinks in the previous 90 days or $13.9 \pm 5.3$ drinks/day). Alcohol-dependent subjects were abstinent $41.1 \pm 16.4$ days (19 to 68 days) at the time of their first study session.

\section{Urinary Free Cortisol (see Table 3)}

There was not a significant difference in urinary free cortisol between the alcohol-dependent and control groups.

\section{Secretory Characteristics and Mean Basal Concentrations of ACTH and Cortisol (see Table 2 and Figs. 1 and 2)}

Secretory characteristics were determined by SBPP, an algorithm to assess pulsatility that allows for a changing baseline. One healthy control was excluded from the SBPP analy- sis because of several missing measurements. As a reasonable fit was not detected for ACTH profile of 2 patients, these subjects were also excluded from the SBPP analysis on ACTH. Neither ACTH nor cortisol pulsatile characteristics significantly differed between the 2 groups. As expected, the Time $\times$ Group ANOVA analyses showed a significant within group Time effect $\left[\mathrm{F}_{(3,45)}=72.1, \quad p<0.0001\right]$ for ACTH 3-hour blocks. However, there was no Group $\left[\mathrm{F}_{(1,14)}=0.33, p=0.58\right]$ or Group $\times$ Time $\left[\mathrm{F}_{(3,42)}=0.15\right.$, $p=0.93]$ effect. Similarly, there was a significant Time effect $\left[\mathrm{F}_{(3,42)}=98.9, p<0.0001\right]$ but not a Group $\left[\mathrm{F}_{(1,14)}=1.97\right.$, $p=0.18]$ or Group $\times$ Time $\left[\mathrm{F}_{(3,42)}=0.60, p=0.62\right]$ effect for the cortisol 3-hour blocks. Exploratory post hoc assessment (Table 2) of each 3-hour block period revealed a nonsignificant decrease $(p<0.07)$ in cortisol during the 0200-0500 hour block in alcohol-dependent subjects compared to the controls but no other significant group differences for either hormone (see Fig. 2).

\section{Cosyntropin Stimulation Test (see Table 3 and Fig. 3)}

The net peak cortisol response in the control female group was similar to that previously observed in male controls (Adinoff et al., 2005a) (men: $8.7 \pm 3.5$, women: $7.8 \pm 1.7$; $t=0.73, \mathrm{df}=18, p=0.47$ ). The cortisol response to lowdose cosyntropin, as described by net integrated response (integrated response from 0800 to 1000 hour minus the mean basal concentration from 0730 to 0800 ), was not significantly different between the 2 groups. As the net integrated response in 4 subjects ( 3 controls, 1 patient) was negative as a result of their final cortisol concentrations decreasing to less than basal measures, the integrated net response from 0800 to 0900 hour (during which cortisol levels remained above basal

Table 2. ACTH and Cortisol (mean \pm SD) Pulsatile Characteristics as Determined by Smoothing Baseline Pulse Pulses (SBPP) Analysis and Mean ACTH and Cortisol Concentrations Over 12 Hours (2000 to 0800 Hour) and in 3-Hour Blocks. See Text for Description of SBPP Measures.

\begin{tabular}{|c|c|c|c|c|c|c|}
\hline & Healthy controls & Alcohol-dependent & T-stat & df & $p$ Value & Effect size \\
\hline \multicolumn{7}{|c|}{ ACTH pulsatile characteristics } \\
\hline Total probability & $20.3 \pm 12.5$ & $13.0 \pm 12.4$ & 1.05 & 12 & 0.32 & 0.6 \\
\hline Adjusted input & $138.7 \pm 81.8$ & $100.2 \pm 96.1$ & 0.76 & 12 & 0.47 & 0.4 \\
\hline Number of inputs & $17.5 \pm 13.7$ & $12.4 \pm 11.6$ & 0.74 & 12 & 0.47 & 0.4 \\
\hline Average baseline & $14.6 \pm 4.2$ & $20.5 \pm 9.3$ & -1.34 & 12 & 0.24 & 0.9 \\
\hline Mean amplitude & $7.0 \pm 3.6$ & $9.3 \pm 3.5$ & -1.15 & 12 & 0.28 & 0.7 \\
\hline \multicolumn{7}{|c|}{ Mean ACTH concentrations (pg/ml) } \\
\hline 2000-2300 hour & $14.0 \pm 4.8$ & $15.8 \pm 5.7$ & -0.75 & 14 & 0.46 & \\
\hline 2300-0200 hour & $17.6 \pm 5.4$ & $19.4 \pm 8.7$ & -0.50 & 14 & 0.63 & \\
\hline 0200-0500 hour & $24.5 \pm 3.2$ & $27.0 \pm 9.3$ & -0.68 & 14 & 0.52 & \\
\hline 0500-0800 hour & $29.9 \pm 5.8$ & $32.8 \pm 9.2$ & -0.77 & 14 & 0.46 & \\
\hline \multicolumn{7}{|c|}{ Cortisol pulsatile characteristics } \\
\hline Total probability & $14.1 \pm 6.7$ & $16.3 \pm 8.0$ & -0.58 & 14 & 0.57 & 0.3 \\
\hline Adjusted input & $36.7 \pm 14.3$ & $33.3 \pm 11.4$ & 0.74 & 14 & 0.61 & 0.3 \\
\hline Number of inputs & $12.7 \pm 5.7$ & $14.1 \pm 6.5$ & -0.44 & 14 & 0.67 & 0.2 \\
\hline Average baseline & $2.3 \pm 1.8$ & $2.0 \pm 1.3$ & 0.38 & 14 & 0.71 & 0.2 \\
\hline Mean amplitude & $3.0 \pm 1.2$ & $2.3 \pm 1.0$ & 1.35 & 14 & 0.20 & 0.6 \\
\hline \multicolumn{7}{|c|}{ Mean cortisol concentrations $(\mu \mathrm{g} / \mathrm{dl})$} \\
\hline 2000-2300 hour & $2.6 \pm 1.1$ & $2.5 \pm 1.1$ & 0.31 & 14 & 0.76 & \\
\hline 2300-0200 hour & $3.5 \pm 2.3$ & $2.3 \pm 0.9$ & 1.40 & 14 & 0.19 & \\
\hline 0200-0500 hour & $7.7 \pm 1.7$ & $5.9 \pm 2.1$ & 1.93 & 14 & 0.07 & \\
\hline 0500-0800 hour & $11.4 \pm 1.4$ & $10.1 \pm 2.7$ & 1.33 & 14 & 0.21 & \\
\hline
\end{tabular}



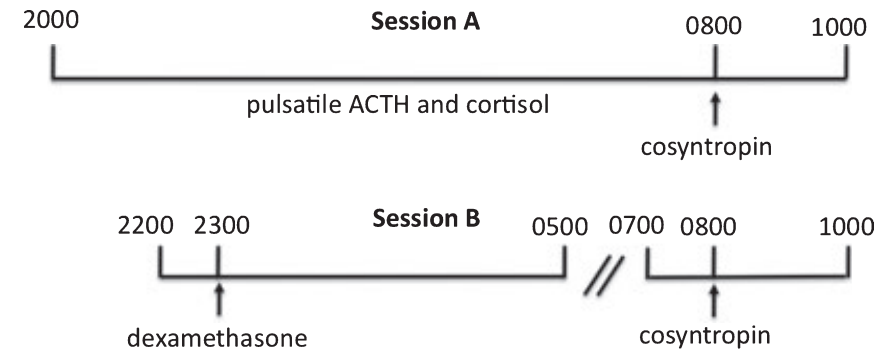

Fig. 1. Study design of pulsatility and cosyntropin/dexamethasone challenges. Session A: 24-hour urinary free cortisol (not shown) was obtained from 2000 to 2000 hour prior to the pulsatility measures. ACTH and cortisol concentrations were obtained from 2000 to 0800 to determine baseline pulsatile characteristics. Cosyntropin $(0.03 \mu \mathrm{g} / \mathrm{kg})$ was administered at 0800 to assess adrenocortical sensitivity. Session B: Dexamethasone (8 mg IV) was administered at 2300 to suppress endogenous ACTH secretion and to assess pituitary corticotroph sensitivity. Cosyntropin was administered at 0800 to assess adrenocortical sensitivity in the relative absence of endogenous ACTH. Sessions were counter-balanced. Blood draws for ACTH and cortisol concentrations were obtained every 10 minutes during all the intervals noted, except blood draws were increased to every 5 minutes for 1 hour after cosyntropin infusions.

concentrations) was also determined. There was not a between-group difference between net integrated response during this 1-hour period (control subjects $=3099.0 \pm$ $618.3 \mu \mathrm{g} / \mathrm{dl} / 1$ hour; patients: $4111.78 \pm 581.3 \mu \mathrm{g} / \mathrm{dl} / 1 \mathrm{~h}$; $t=0.93, \mathrm{df}=15, p=0.36$ ). The net cortisol peak response to cosyntropin was somewhat higher in the control group relative to the patients, although this difference did not reach statistical significance $(p<0.08)$. As seen in Fig. 3, cortisol concentrations were somewhat higher (from 15 to 25 minutes) following cosyntropin administration in the control relative to the patient group, although the early (5 to 10 minutes) and later (30 to 120 minutes) time points were nearly identical between groups.

\section{Dexamethasone Suppression of ACTH and Cortisol (Table 3 and Fig. 4)}

Dexamethasone $8 \mathrm{mg}$ was administered intravenously at 2300 hour, and the ACTH and cortisol response was assessed from 2300 to 0500 hour. ACTH was somewhat lower in the control group relative to the patients, although this difference did not reach statistical significance. There were no betweengroup differences in cortisol following dexamethasone. Both groups suppressed ACTH concentrations to levels approximately $50 \%$ of those observed during peak hours and mean cortisol concentrations were below $3.2 \mu \mathrm{g} / \mathrm{dl}$ (mean) from 0230 hour until the administration of cosyntropin at 0800 hour.

\section{Dexamethasone + Cosyntropin Stimulation Test (see Table 3 and Fig. 5)}

Cosyntropin $(0.01 \mu \mathrm{g} / \mathrm{kg})$ was administered following dexamethasone suppression of endogenous ACTH. One healthy control was excluded because of missing data. Just

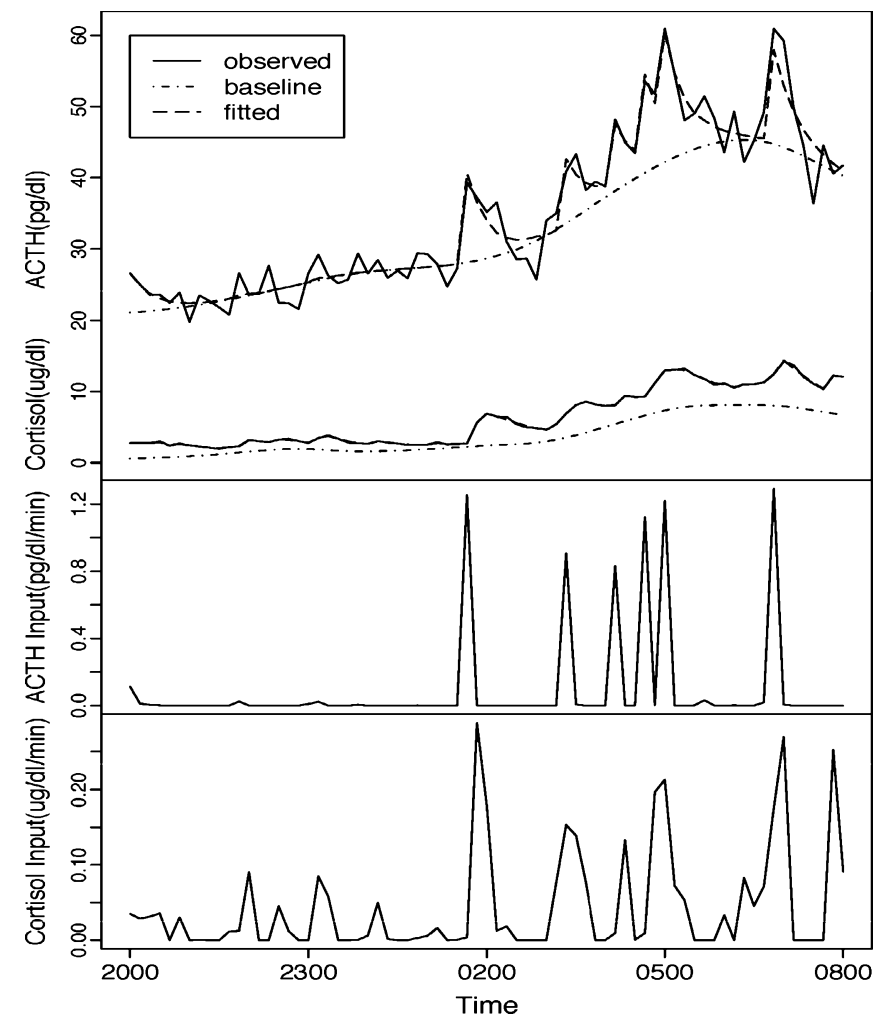

Fig. 2. This figure illustrates the Smoothing Baseline Pulse Pulses (SBPP) of 12 hours (2000 to 0800 hour) of ACTH and cortisol data from a single subject. SBPP decomposes the hormone profile (solid line in top panel) into a slowly changing baseline (dotted line in top panel) and fast changing pulses (bottom two panels). (A) The original data (solid line), its fitted values (dashed line) and the estimated baseline (dotted line). ACTH measures are on top and cortisol measures on the bottom. (B) The estimated pulse inputs of ACTH. (C) The estimated pulse inputs of cortisol.

prior to cosyntropin administration, ACTH and cortisol mean concentrations ( 0730 to 0800 hour) were similar between the groups. The net peak cortisol response in the control female group was nearly identical to that previously observed in male controls (Adinoff et al., 2005a) (men: $15.8 \pm 2,9$, women: $16.1 \pm 3.0 ; t=0.20, \mathrm{df}=18, p=$ 0.84). The net integrated and net peak cortisol responses to the cosyntropin challenge were nearly identical between the alcohol-dependent and control women.

\section{Correlations Between Neuroendocrine Measures and Drinking and Smoking History}

The relationship between drinking history (i.e., drinks in previous ninety days, lifetime drinks, days abstinent) and neuroendocrine measures that demonstrated a trend toward statistical significance between groups (mean basal cortisol 0200-0500 hour, mean cortisol peak response following cosyntropin) were considered. Correlations between neuroendocrine variables and pack years smoking were also assessed. There were no significant correlations observed. 
Table 3. Urinary Free Cortisol and Basal and Stimulated ACTH and Cortisol Response to Cosyntropin ( $0.01 \mu \mathrm{g} / \mathrm{kg}$ intravenously), Pituitary-Adrenal Response to Intravenously Dexamethasone (8 mg intravenously), and Cosyntropin Following Dexamethasone Pretreatment (mean \pm SD)

\begin{tabular}{|c|c|c|c|c|c|c|}
\hline & $\begin{array}{l}\text { Healthy } \\
\text { controls }\end{array}$ & Alcohol-dependent & t stat & df & $p$ Value & $\begin{array}{l}\text { Effect } \\
\text { size }\end{array}$ \\
\hline 24-hour urinary free cortisol (g/24 hour) & $69.0 \pm 38.7$ & $63.9 \pm 29.8$ & 0.27 & 13 & 0.79 & \\
\hline \multicolumn{7}{|l|}{ Cosyntropin stimulation test without dexamethasone } \\
\hline Mean basal cortisol ( $\mu \mathrm{g} / \mathrm{dl})$ (0730-0800 hour) & $10.4 \pm 2.9$ & $10.9 \pm 2.4$ & 0.367 & 15 & 0.72 & 0.2 \\
\hline Net AUC of cortisol following cosyntropin ( $\mu \mathrm{g} / \mathrm{dl} .2$ hour) & $249.5 \pm 265.0$ & $199.8 \pm 239.7$ & 0.396 & 15 & 0.69 & 0.2 \\
\hline Net peak cortisol response following cosyntropin $(\mu \mathrm{g} / \mathrm{dl})$ & $7.8 \pm 1.7$ & $6.3 \pm 1.7$ & 1.846 & 15 & 0.08 & 0.4 \\
\hline $\begin{array}{l}\text { Mean cortisol response following DEX }(\mu \mathrm{g} / \mathrm{dl}) \\
(2330-0500 \text { hour })\end{array}$ & $2.1 \pm 1.7$ & $1.4 \pm 0.2$ & 1.114 & 15 & 0.28 & 0.6 \\
\hline Mean basal ACTH (pg/ml) (0730-0800 hour) & $8.3 \pm 3.9$ & $11.1 \pm 4.8$ & 1.267 & 14 & 0.22 & 0.7 \\
\hline Mean basal cortisol ( $\mu \mathrm{g} / \mathrm{dl})$ (0730-0800 hour) & $1.1 \pm 0.3$ & $1.3 \pm 0.7$ & 0.738 & 14 & 0.47 & 0.4 \\
\hline $\begin{array}{l}\text { Net AUC of cortisol response following cosyntropin } \\
\text { ( } \mu \mathrm{g} / \mathrm{dl} .2 \text { hour })\end{array}$ & $957.4 \pm 258.7$ & $994.4 \pm 131.7$ & 0.316 & 14 & 0.75 & 0.2 \\
\hline
\end{tabular}

AUC, area under the curve method.

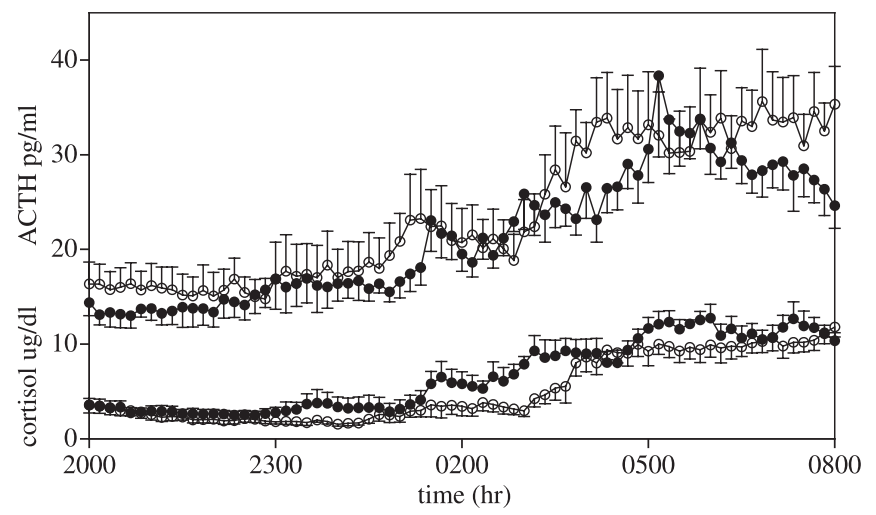

Fig. 3. Mean \pm SEM of ACTH (upper) and cortisol (lower) basal concentrations. Measures between 2000 and 0800 hours reflect basal concentrations. Healthy control subjects are closed circles; alcohol-dependent subjects are open circles.

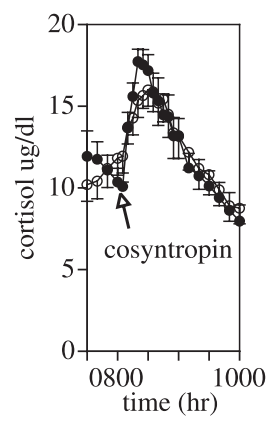

Fig. 4. Mean \pm SEM of cortisol concentrations. Cosyntropin $(0.01 \mu \mathrm{g} / \mathrm{kg}$ intravenously) was administered at 0800 hour. Healthy control subjects are closed circles; alcohol-dependent subjects are open circles.

\section{DISCUSSION}

These findings suggest that adrenocortical reactivity, pituitary corticotroph sensitivity, and ACTH and glucocorticoid
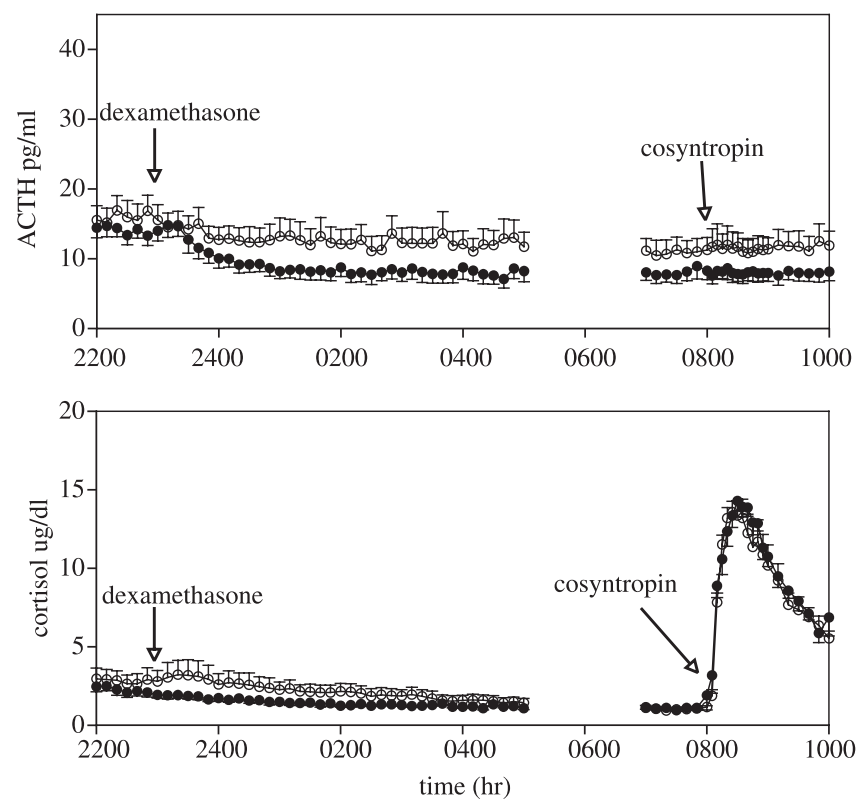

Fig. 5. Mean \pm SEM of ACTH (upper panel) and cortisol (lower panel) concentrations. Dexamethasone (8 $\mathrm{mg}$ intravenously) was administered at 2300 hour. Cosyntropin $(0.01 \mu \mathrm{g} / \mathrm{kg}$ intravenously) was administered at 0800 hour. Healthy control subjects are closed circles; alcohol-dependent subjects are open circles.

circadian rhythmicity does not significantly differ between abstinent alcohol-dependent women and healthy control women. Although there was a suggestion of a blunted cortisol peak response in the alcohol-dependent group following cosyntropin, neither the net integrated response to cosyntropin nor the peak or integrated cortisol response to cosyntropin following dexamethasone, more rigorous assessments of adrenocortical reactivity, significantly differed between groups. These findings are notably different from those previously reported in male alcohol-dependent subjects using a nearly identical paradigm (Adinoff et al., 
2005a). The primary shared finding in both male (Adinoff et al., 2005a) and female alcohol-dependent subjects were somewhat lower basal cortisol concentrations in the early morning (0200 to 0500 hour). Even though statistically marginal, however, relatively higher basal ACTH concentrations and lower cortisol concentrations in the early morning hours and somewhat higher post-dexamethasone ACTH concentrations in alcohol-dependent women, relative to controls, could suggest a disruption in feed forward and feedback mechanisms.

The small $\mathrm{n}$ of our sample was a major limiting factor. This limitation was mitigated, at least in part, by the carefully selected subject sample (no lifetime history of Axis I disorders occurring in the absence of recent or ongoing alcohol use, no recent use of psychotropics, a confined period of abstinence, no ongoing use of oral contraceptives, premenopausal women studied during the early follicular phase of their menstrual cycle), the heavy drinking in all patients (at least 450 drinks in the 90 days prior to admission), frequent plasma sampling, and the use of multiple endocrine measures. The 2 groups were also age-matched, although they differed in education levels and family history of other psychiatric disorders (the latter was cause for exclusion only in the controls).

Further limitations included: (i) diurnal measures using only 12 hours of sampling. This time course, however, includes the period most likely to demonstrate pathological alterations (during peak activity). (ii) The use of manual blood withdrawal techniques may have disrupted sleep patterns, effecting ACTH and cortisol pulsatility. Although efforts were taken to avoid awakening subjects, we did not obtain objective measures of sleep disruption or potential mood disturbances that could have resulted. (iii) The administration of pituitary-adrenal challenges are typically performed in the late afternoon or early evening, although Dickstein and colleagues (1997) has reported that the cortisol response to a submaximal dose of cosyntropin is similar at 0800 and 1600 hour. (iv) In order to study all women during the same time of their menstrual phase, the alcohol-dependent women were studied following a somewhat longer period of abstinence (41.4 \pm 16.4 days) than the previously reported male subjects $(31.0 \pm 4.0$ days). (v) Alcohol-dependent women smoked significantly more than their control counterparts. This confound generally plagues most clinical laboratory research of substance abusers, as most substance-dependent patients smoke and most healthy volunteers without medical or psychiatric morbidity, particularly in the age range of our patient population, do not. Anthenelli and colleagues (2001) has reported that nicotine dependence does not account for the HPA axis alterations observed in alcohol-dependent men. Although the acute nicotine use stimulates the HPA axis (Mendelson et al., 2008), our nicotine-dependent subjects had not smoked for several hours prior to study initiation and nicotine patches were placed well before study onset. Although subjects were not assessed for nicotine withdrawal symptoms, recently abstinent smokers show stable cortisol circadian concentrations when placed on a nicotine patch (Teneggi et al., 2002).

Despite the potential confounds, many of the findings were unequivocally negative. For instance, the glucocorticoid response to cosyntropin following dexamethasone was nearly identical in both groups, and effect sizes for our primary measures (net AUC cortisol response) were small. Approximately 400 subjects per group would have been required to detect significant group differences in the net AUC cortisol following either cosyntropin or cosyntropin following dexamethasone. In contrast, this paradigm revealed one of the strongest group differences in the male populations (Adinoff et al., 2005a). In addition, the numerical elevation in ACTH response to dexamethasone in the alcohol-dependent women (suggesting a muted pituitary corticotroph sensitivity to glucocorticoid feedback) was also in the opposite direction to the increased pituitary corticotroph sensitivity (i.e., decreased ACTH response) observed in the male patient population.

The absence of glucocorticoid hyporeactivity in the alcohol-dependent women was unexpected. As noted, adrenocortical hyporeactivity has been previously reported in male alcohol-dependent subjects. In addition, Heim and colleagues (2001) has observed that nondepressed women with childhood abuse show a blunted glucocorticoid response to cosyntropin compared to nonabused control group. Although we did not assess abuse in our study population, previous work in this area would suggest that the alcohol-dependent women had experienced significantly more childhood abuse than our healthy control group (Felitti et al., 1998; Kendler et al., 2000; Sher et al., 1997). Nevertheless, neither the chronic use of alcohol nor presumed trauma history appeared to result in altered adrenocortical reactivity.

Most studies demonstrating a blunted cortisol response in alcohol-dependent men have used stimuli that induce glucocorticoid release indirectly through pituitary ACTH (Adinoff et al., 2005b; Bailly et al., 1989; Chalmers et al., 1978; Coiro et al., 2007; Coiro and Vescovi, 1999; Costa et al., 1996; Errico et al., 1993; Lovallo et al., 2000; Margraf et al., 1967; Merry and Marks, 1972; Vescovi et al., 1997). As some (Coiro et al., 2007; Coiro and Vescovi, 1999; Vescovi et al., 1997), but not all (Adinoff et al., 2005b; Bailly et al., 1989), of these paradigms also report a concurrent blunting of ACTH stimulation, the apparent adrenocortical hyporesponsivity may be a consequence of an attenuated upper-level stimulus. Other studies do not report differences in glucocorticoid responsiveness in alcohol-dependent subjects even in the presence of a muted ACTH response (Adinoff et al., 1990; Inder et al., 1995) or an exaggerated, not subdued, glucocorticoid response is observed (Anthenelli et al., 2001; George et al., 1994). In fact, Brady and colleagues (2006) reported a blunted ACTH response in alcohol-dependent men and women following a cold pressor task that was more marked (albeit nonsignificantly) in women than men. Cortisol concentrations, however, showed no between-group (alcohol-dependent vs. controls) or gender (men vs. women) differences (Brady et al., 2006). Using only single basal measures during abstinence, 
Gianoulakis et al. (2003) reported that plasma cortisol was higher in 30 to $60 \mathrm{y} / \mathrm{o}$ alcohol-dependent men in treatment relative to sex-matched nondrinkers, whereas female alcoholdependent women did not significantly differ from their nondrinking comparison group.

Alcohol-dependent men and women differ in their genetic, social and personality vulnerabilities to alcohol dependence, their initial onset of disease progression, and the long-term consequences of heavy alcohol use (Nolen-Hoeksema and Hilt, 2006; Walitzer and Dearing, 2006). The unanticipated findings in the present study emphasize the importance in assessing the neurobiological disruptions in both male and female alcohol-dependent subjects that may underlie these clinical differences. Of potential relevance is evidence suggesting that long-acting naltrexone is effective in preventing alcohol relapse in men and not women (Garbutt et al., 2005). A possible mechanism of naltrexone is its disinhibiting effects upon the HPA axis, thus mitigating the pituitary-adrenal suppression that accompanies chronic alcohol dependence in men (Adinoff et al., 1998). If alcohol-dependent women do not suffer from a similar deficit in the HPA axis, then naltrexone may, at least in part, lose some of its effectiveness. Future studies should continue to explore HPA deficits in female alcohol-dependent women, including higher level (i.e., prefrontal cortex, limbic system, hypothalamus, pituitary) disruptions, the relationship of HPA alterations to prior trauma and adverse life events, the predictive effects of HPA axis alterations and relapse, and the effects of pharmacological interventions upon the HPA axis.

\section{ACKNOWLEDGMENTS}

The authors gratefully acknowledge the assistance of Homeward Bound, Inc. and Dallas VA Substance Abuse Team in the recruitment and clinical care of patients and the UT Southwestern GCRC staff for their excellent patient care and meticulous attention to research protocol. This work was funded by \#AA1570 (National Institute on Alcohol Abuse and Alcoholism) and M01-RR00633 (USPHS).

\section{REFERENCES}

Adinoff B, Iranmanesh A, Veldhuis J, Fisher L (1998) Disturbances of the stress response: the role of the HPA axis during alcohol withdrawal and abstinence. Alcohol Health Res World 22(1):67-72.

Adinoff B, Krebaum SR, Chandler PA, Ye W, Brown MB, Williams MJ (2005a) Dissection of hypothalamic-pituitary-adrenal axis pathology in 1-month-abstinent alcohol-dependent men, part 1: adrenocortical and pituitary glucocorticoid responsiveness. Alcohol Clin Exp Res 29(4): $517-527$.

Adinoff B, Krebaum SR, Chandler PA, Ye W, Brown MB, Williams MJ (2005b) Dissection of hypothalamic-pituitary-adrenal axis pathology in 1-month-abstinent alcohol-dependent men, part 2: response to ovine corticotropin-releasing factor and naloxone. Alcohol Clin Exp Res 29(4):528 537.

Adinoff B, Martin PR, Bone GHA, Eckardt MJ, Roehrich L, George DT, Moss HB, Eskay R, Linnoila M, Gold PW (1990) Hypothalamic-pituitaryadrenal axis functioning and cerebrospinal fluid corticotropin releasing hormone and corticotropin levels in alcoholics after recent and long-term abstinence. Arch Gen Psychiatry 47:325-330.

Adinoff B, Risher-Flowers D, De Jong J, Ravitz B, Bone GHA, Nutt DJ, Roehrich L, Martin PR, Linnoila M (1991) Disturbances of hypothalamicpituitary-adrenal axis functioning during ethanol withdrawal in six men. Am J Psychiatry 148:1023-1025.

Adinoff B, Ruether K, Krebaum S, Iranmanesh A, Williams MJ (2003) Increased salivary cortisol concentrations during chronic alcohol intoxication in a naturalistic clinical sample of men. Alcohol Clin Exp Res 27(9):1420-1427.

Anthenelli RM, Maxwell RA, Geracioti TDJ, Hauger R (2001) Stress hormone dysregulation at rest and after serotonergic stimulation among alcohol-dependent men with extended abstinence and controls. Alcohol Clin Exp Res 25(5):692-703.

Bailly D, Dewailly D, Beuscart R, Couplet G, Dumont P, Racadot A, Fossati P, Parquet PJ (1989) Adrenocorticotropin and cortisol responses to ovine corticotropin-releasing factor in alcohol dependence disorder. Preliminary report. Horm Res 31(1-2):72-75.

Beck AT, Ward CH, Mendelson M, Mock J, Erbaugh J (1979) An inventory for measuring depression. Arch Gen Psychiatry 4:561-571.

Brady KT, Grice DE, Dustan L, Randall C (1993) Gender differences in substance use disorders. Am J Psychiatry 150(11):1707-1711.

Brady KT, Waldrop AE, McRae AL, Back SE, Saladin ME, Upadhyaya HP, Anton RF, Randall PK (2006) The impact of alcohol dependence and posttraumatic stress disorder on cold pressor task response. J Stud Alcohol 67(5):700-706.

Chalmers RJ, Bennie EH, Johnson RH, Masterton G (1978) Growth hormone, prolactin, and corticosteroid responses to insulin hypoglycaemia in alcoholics. Br Med J 1(6115):745-748.

Coiro V, Casti A, Jotti GS, Rubino P, Manfredi G, Maffei ML, Melani A, Volta E, Chiodera P (2007) Adrenocorticotropic hormone/cortisol response to physical exercise in abstinent alcoholic patients. Alcohol Clin Exp Res 31(5):901-906.

Coiro V, Vescovi PP (1999) Effect of cigarette smoking on ACTH/cortisol secretion in alcoholic after short- and medium-term abstinence. Alcohol Clin Exp Res 23(9):1515-1518.

Costa A, Bono G, Martignoni E, Merlo P, Sances G, Nappi G (1996) An assessment of hypothalamo-pituitary-adrenal axis functioning in nondepressed, early abstinent alcoholics. Psychoneuroendocrinology 21(3):263275.

Dickstein G, Spigel D, Arad E, Shechner C (1997) One microgram is the lowest ACTH dose to cause a maximal cortisol response. There is no diurnal variation of cortisol response to submaximal ACTH stimulation. Eur $\mathbf{J}$ Endocrinol 137(2):172-175.

Ehrenreich H, Schuck J, Stender N, Pilz J, Gefeller O, Schilling L, Poser W, Kaw S (1997) Endocrine and hemodynamic effects of stress versus systemic $\mathrm{CRF}$ in alcoholics during early and medium term abstinence. Alcohol Clin Exp Res 21(7):1285-1293.

Errico AL, Parsons OA, King AC, Lovallo WR (1993) Attenuated cortisol response to biobehavioral stressors in sober alcoholics. J Stud Alcohol 54(4):393-398.

Felitti VJ, Anda RF, Nordenberg D, Williamson DF, Spitz AM, Edwards V, Koss MP, Marks JS (1998) Relationship of childhood abuse and household dysfunction to many of the leading causes of death in adults. The Adverse Childhood Experiences (ACE) Study. Am J Prev Med 14(4):245-258.

First MH, Spitzer RL, Gibbon M, Williams JBW (1996) Structured Clinical Interview for DSM-IV axis I Disorders - Patient Edition (SCID-I/P, Version 2.0). Biometrics Research Department, New York State Psychiatric Institute, NY.

Garbutt JC, Kranzler HR, O'Malley SS, Gastfriend DR, Pettinati HM, Silverman BL, Loewy JW, Ehrich EW (2005) Efficacy and tolerability of long-acting injectable naltrexone for alcohol dependence: a randomized controlled trial. JAMA 293(13):1617-1625.

George DT, Lindquist T, Alim T, Flood M, Eckardt MJ, Linnoila M (1994) Abstinent alcoholics exhibit an exaggerated stress response to 2-deoxy-Dglucose challenge. Alcohol Clin Exp Res 18(3):685-691. 
Gianoulakis C, Dai X, Brown T (2003) Effect of chronic alcohol consumption on the activity of the hypothalamic-pituitary-adrenal axis and pituitary beta-endorphin as a function of alcohol intake, age, and gender. Alcohol Clin Exp Res 27(3):410-423.

Guo W, Wang Y, Brown MB (1999) A signal extraction approach to modeling hormone time series with pulses and a changing baseline. J Am Stat Assoc 94(447):746-756.

Heim C, Newport DJ, Bonsall R, Miller AH, Nemeroff CB (2001) Altered pituitary-adrenal axis responses to provocative challenge tests in adult survivors of childhood abuse. Am J Psychiatry 158(4):575-581.

Inder WJ, Joyce PR, Ellis MJ, Evans MJ, Livesey JH, Donald RA (1995) The effects of alcoholism on the hypothalamic-pituitary-adrenal axis: interaction with endogenous opioid peptides. Clin Endocrinol (Oxf) 43(3):283-290.

Iranmanesh A, Veldhuis JD, Johnson ML, Lizarralde G (1989) 24-hour pulsatile and circadian patterns of cortisol secretion in alcoholic men. J Androl 10:54-63.

Junghanns K, Backhaus J, Tietz U, Lange W, Bernzen J, Wetterling T, Rink L, Driessen M (2003) Impaired serum cortisol stress response is a predictor of early relapse. Alcohol Alcohol 38(2):189-193.

Kandel D, Chen K, Warner LA, Kessler RC, Grant B (1997) Prevalence and demographic correlates of symptoms of last year dependence on alcohol, nicotine, marijuana and cocaine in the U.S. population. Drug Alcohol Depend 44(1):11-29.

Keedwell PA, Poon L, Papadopoulos AS, Marshall EJ, Checkley SA (2001) Salivary cortisol measurements during a medically assisted alcohol withdrawal. Addict Biol 6(3):247-256.

Kendler KS, Bulik CM, Silberg J, Hettema JM, Myers J, Prescott CA (2000) Childhood sexual abuse and adult psychiatric and substance use disorders in women: an epidemiological and cotwin control analysis. Arch Gen Psychiatry 57(10):953-959.

Kiefer F, Jahn H, Otte C, Naber D, Wiedemann K (2006) Hypothalamicpituitary-adrenocortical axis activity: a target of pharmacological anticraving treatment? Biol Psychiatry 60(1):74-76.

Kirschbaum C, Kudielka BM, Gaab J, Schommer NC, Hellhammer DH (1999) Impact of gender, menstrual cycle phase, and oral contraceptives on the activity of the hypothalamus-pituitary-adrenal axis. Psychosom Med 61(2):154-162.

Knudsen GM, Christensen H, Berild D, Melgaard B, Kirkegaard C, Hasselbalch H (1987) Discordance between the cortisol response to insulinhypoglycemia and 30-minute ACTH stimulation test in chronic alcoholic men. Alcohol Clin Exp Res 11(4):323-325.

Lovallo WR, Dickensheets SL, Myers DA, Thomas TL, Nixon SJ (2000) Blunted stress cortisol response in abstinent alcoholic and polysubstanceabusing men. Alcohol Clin Exp Res 24(5):651-658.

Margraf HW, Moyer CA, Ashford LE, Lavalle LW (1967) Adrenocortical function in alcoholics. J Surg Res 7(2):55-62.
Mendelson JH, Goletiani N, Sholar MB, Siegel AJ, Mello NK (2008) Effects of smoking successive low- and high-nicotine cigarettes on hypothalamicpituitary-adrenal axis hormones and mood in men. Neuropsychopharmacology 33(4):749-760.

Mendelson JH, Ogata M, Mello NK (1971) Adrenal function and alcoholism I. serum cortisol. Psychosom Med 33:145-157.

Merry J, Marks V (1972) The effect of alcohol, barbiturate, and diazepam on hypothalamic, pituitary/adrenal function in chronic alcoholics. The Lancet 2:990-992.

Miller WR, Tonigan JS, Longabaugh R (1995) The Drinker Inventory of Consequences (DrlnC) An Instrument for Assessing Adverse Consequences of Alcohol Abuse. National Institutes of Health, Rockville, MD.

Munro CA, Oswald LM, Weerts EM, McCaul ME, Wand GS (2005) Hormone responses to social stress in abstinent alcohol-dependent subjects and social drinkers with no history of alcohol dependence. Alcohol Clin Exp Res 29(7):1133-1138.

Najavits LM, Weiss RD, Shaw SR (1997) The link between substance abuse and posttraumatic stress disorder in women. A research review. Am J Addict 6(4):273-283.

Nolen-Hoeksema S, Hilt L (2006) Possible contributors to the gender differences in alcohol use and problems. J Gen Psychol 133(4):357-374.

Shalev I, Lerer E, Israel S, Uzefovsky F, Gritsenko I, Mankuta D, Ebstein RP, Kaitz M (2008) BDNF Val66Met polymorphism is associated with HPA axis reactivity to psychological stress characterized by genotype and gender interactions. Psychoneuroendocrinology 34(3):382-388.

Sher KJ, Gershuny BS, Peterson L, Raskin G (1997) The role of childhood stressors in the intergenerational transmission of alcohol use disorders. J Stud Alcohol 58(4):414-427.

Sobell MB, Sobell LC (1978) Behavioral Treatment of Alcohol Problems. Plenum Press, NewYork.

Stokes PE (1973) Adrenocortical activation in alcoholics during chronic drinking. Ann NY Acad Sci 215:77-83.

Teneggi V, Tiffany ST, Squassante L, Milleri S, Ziviani L, Bye A (2002) Smokers deprived of cigarettes for $72 \mathrm{~h}$ : effect of nicotine patches on craving and withdrawal. Psychopharmacology (Berl) 164(2):177-187.

Uhart M, Chong RY, Oswald L, Lin PI, Wand GS (2006) Gender differences in hypothalamic-pituitary-adrenal (HPA) axis reactivity. Psychoneuroendocrinology 31(5):642-652.

Vescovi PP, DiGennaro C, Coiro V (1997) Hormonal (ACTH, cortisol, betaendorphin, and met-enkephalin) and cardiovascular responses to hyperthermic stress in chronic alcoholics. Alcohol Clin Exp Res 21(7):1195-1198.

Walitzer KS, Dearing RL (2006) Gender differences in alcohol and substance use relapse. Clin Psychol Rev 26(2):128-148.

Wand GS, Dobs AS (1991) Alterations in the hypothalamic-pituitary-adrenal axis in actively drinking alcoholics. J Clin Endocrinol Metab 72(6):12901295. 